\title{
A swelling of the maxilla: a case report and differential diagnosis
}

\author{
Puneet Bhargava', Saba Khan ${ }^{1}$, Rohit Sharma ${ }^{1}$, Khalid Agwani ${ }^{2}$, Sahil Gupta ${ }^{1}$ \\ ${ }^{I}$ Department of Oral Medicine and Radiology, NIMS Dental College and Hospital, NIMS University, Jaipur, \\ ${ }^{2}$ Department of Oral and Maxillofacial Surgery, Darshan Dental College, Udaipur, India
}

\begin{abstract}
J Korean Assoc Oral Maxillofac Surg 2014;40:308-312)
Ossifying fibromas are benign fibro-osseous tumors of mesenchymal origin. Although ossifying fibromas have principally been found in the jaw, they have also been reported in the frontal, ethmoid, sphenoid, and temporal bones, as well as the orbit and anterior cranial fossa. Ossifying fibromas affecting the jaw exhibit variable behaviors ranging from slow growth to occasionally aggressive local destruction. In the present article, we discuss a differential diagnosis considered for maxillary swellings and report a rare case of ossifying fibroma occurring in the maxilla.
\end{abstract}

Key words: Bone neoplasms, Ossifying fibroma, Maxilla, Tomography

[paper submitted 2014. 7. 31 / accepted 2014. 9. 2]

\section{Introduction}

In 1872, Menzel gave the first description of a variant of ossifying fibroma calling it a cemento-ossifying fibroma. In 1927, Montgomery first used the term ossifying fibroma (OF), by which the lesion is currently known ${ }^{1}$. The World Health Organization (WHO) in 1971 classified cementum containing lesions into fibrous dysplasia, OF, cementifying fibroma, and cemento OF. However, the term cemento OF was reduced to OF in 2005 in the new WHO classification ${ }^{2}$.

\section{Case Report}

A 55-year-old female patient visited the dental out patient department with the chief complaint of a swelling in the upper left back teeth region over the past year. The swelling was non tender and initially small but had gradually enlarged to the present size. She had no history of associated pain,

\section{Khalid Agwani}

Department of Oral and Maxillofacial Surgery, Darshan Dental College, Ranakpur Road, Loyara, Udaipur 313001, Rajasthan, India

TEL: +91-8875766866 FAX: +91-1412605050

E-mail:dr.agwani@gmail.com

(c) This is an open-access article distributed under the terms of the Creative Commons Attribution Non-Commercial License (http://creativecommons.org/licenses/by-nc/3.0/), which permits unrestricted non-commercial use, distribution, and reproduction in any medium, provided the original work is properly cited.

Copyright (C) 2014 The Korean Association of Oral and Maxillofacial Surgeons. All rights reserved. discharge, fever or fluctuation in the size, or similar swellings elsewhere in the body. The patient's medical and habit history was non-contributory. All vital signs were within normal limits. Intra-oral examination revealed a large, solitary, well-defined, nodular exophytic growth in the region of the attached gingiva; it was $2.2 \mathrm{~cm}$ in maximum dimension and extended posteriorly distal of tooth 27 involving the tuberosity region. Superioinferiorly it extended from the buccal vestibule extending inferiorly covering the middle $1 / 3$ of crown 27. The color was the same as that of the normal attached gingiva without normal stippling, and the surface was regular and smooth. Palpatory findings revealed non-tender, non-pul-

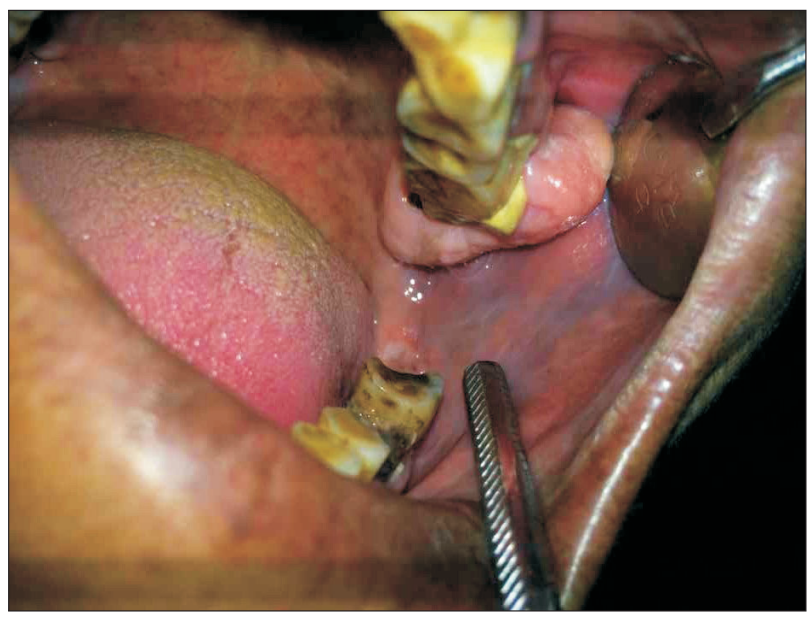

Fig. 1. Nodular exophytic growth in regions 27 and 28. Puneet Bhargava et al: A swelling of the maxilla: a case report and differential diagnosis. J Korean Assoc Oral Maxillofac Surg 2014 


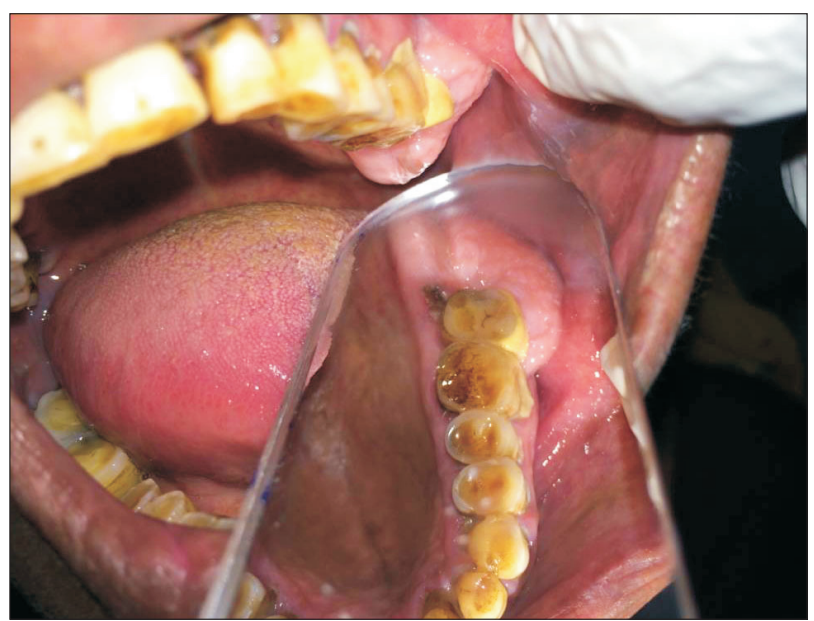

Fig. 2. Buccopalatal extent of the lesion.

Puneet Bhargava et al: A swelling of the maxilla: a case report and differential diagnosis. J Korean Assoc Oral Maxillofac Surg 2014

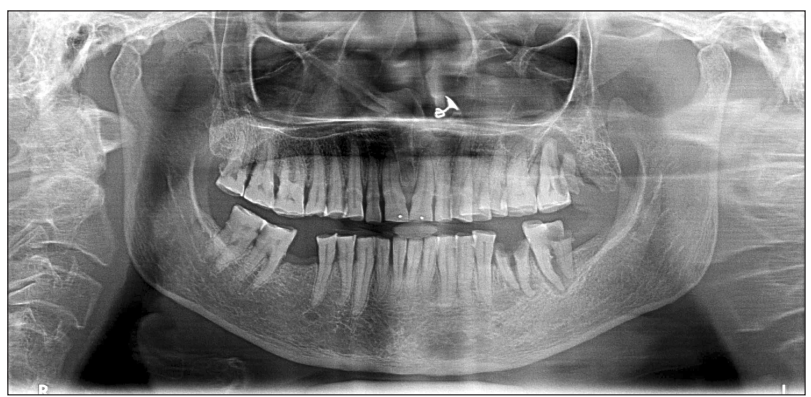

Fig. 3. Orthopantomograph reveals bony overgrowth in the maxillary tuberosity region.

Puneet Bhargava et al: A swelling of the maxilla: a case report and differential diagnosis. J Korean Assoc Oral Maxillofac Surg 2014

satile swelling of bony hard consistency and fluctuancy and compressibility were absent.(Figs. 1,2) The overlying and surrounding mucosa were normal. On probing distal of 27, the suspected root stump of 28 was felt. Hard tissue examination revealed missing 46 , root stump with 36 , and generalized attrition with multiple wear facets.

Orthopantomograph was advised and revealed an area of radiolucency at the periapical regions of 28 and 27 extending distal of 26, which was suggestive of a periapical abscess at 27 and 28 . There was a bony overgrowth in a maxillary tuberosity region showing increased marrow spaces with a fine, wispy, trabecular pattern.(Fig. 3) An axial section from computed tomography at the mid root level of the maxillary arch showed a hyper dense shadow measuring $23.9 \times 13.0 \mathrm{~mm}$ extending distal from 28 and involving the tuberosity region. The Hounsfield unit of the lesion was slightly lower (112240) than the normal trabecular cancellous pattern (326-390), indicating that normal bone was replaced by fibrous connec-

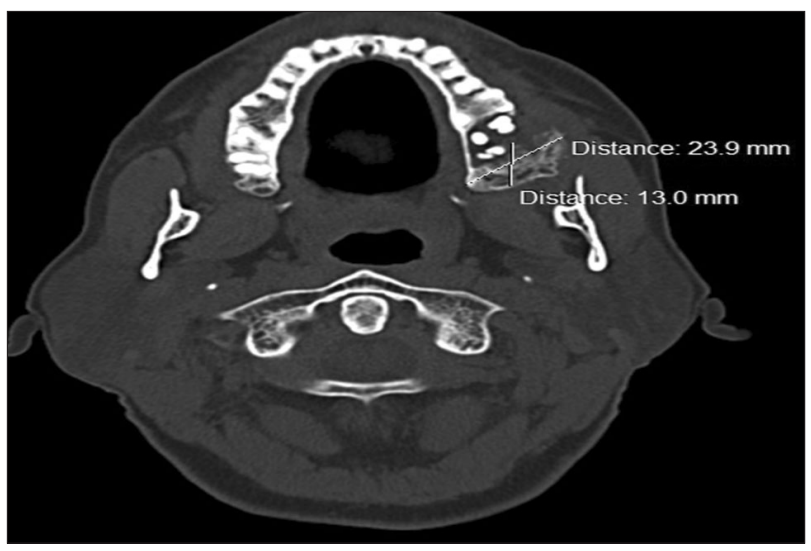

Fig. 4. Axial section of computed tomography at the mid root level of the maxillary arch shows a hyperdense shadow at the tuberosity region.

Puneet Bhargava et al: A swelling of the maxilla: a case report and differential diagnosis. J Korean Assoc Oral Maxillofac Surg 2014

tive tissue suggestive of a fibro osseous lesion. The presence of a hypo dense shadow indicated alveolar bone loss at the periapical area and was suggestive of a periapical abscess at the root stump of 28. Erosion of the buccal cortical plate was also evident.(Fig. 4) An incisional biopsy was done, and the specimen was sent for histopathological examination. The microscopic exam revealed non-keratinised stratified squamous epithelium and peripheral fibroblastic proliferation held tightly in the osteoblastic structures with imperfect ossification and regular trabeculae and fibrous connective tissue in certain places. Encased osteocytes were scattered within the operative specimen. The underlying connective tissue showed collagen fiber bundles, bony trabeculae, extravasated red blood cells, anastamosing trabecular bone with benign fibroblastic proliferation, and islands of bone within fibroblastic stroma.(Figs. 5, 6) Based on the clinical, radiological, and histopathological features, a final diagnosis of OF was made. The patient was scheduled for surgical resection of the lesion and kept on a regular follow-up schedule.

\section{Discussion}

A differential diagnosis for a well-defined maxillary swelling should be achieved based on the anatomical structures present in that location. Some of these structures may be developmental in origin, such as tori or exostosis. Exostosis is seen as a bony hard, smoothly contoured exophytic mass usually present throughout the developmental age ${ }^{3}$. Infectious origin swellings such as periapical abscesses or cysts may involve a tender regional carious or non-vital tooth and 


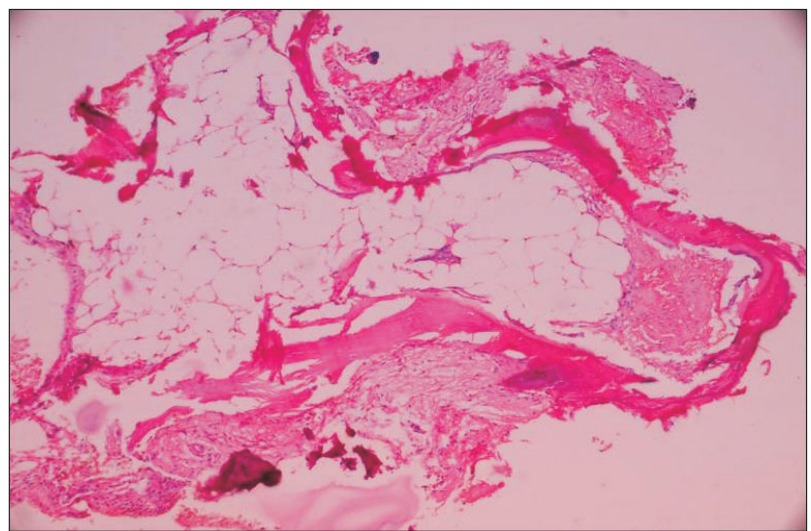

Fig. 5. Histopathology revealed peripheral fibroblastic proliferation, and imperfect ossification (H\&E staining, $\times 40)$.

Puneet Bhargava et al: A swelling of the maxilla: a case report and differential diagnosis. J Korean Assoc Oral Maxillofac Surg 2014

will show signs of inflammation. Radiolucent areas will also be observed in approximation with the apex of the involved teeth, and the swelling will be soft, fluctuant, and painful ${ }^{4}$. Proliferative periostitis due to chronic odontogenic infection beneath the periosteum presents as convex swelling with normal overlying mucosa; it is seen in younger individuals with the inferior border of the mandible below the first molar being the most prevalent site. Radiographically, these lesions present with an onion peel pattern ${ }^{5}$. Swellings due to chronic irritation can be traced back to a history of trauma such as traumatic fibroma or epulis fissuratum or from a sharp tooth, denture margins, or calculus. These are soft to firm, pedunculated, and arise from mucosa or gingiva rather than alveolar bone or jaw ${ }^{6}$. Trauma induced periosteum reactions are seen as smooth, bony hard swellings merging with adjacent bone. The most common salivary gland tumors in the maxilla are pleomorphic adenomas, which appear as a slow growing painless, firm, palpable mass on the palate encased in a pseudo capsule. Conversely, malignant tumors such as adenoid cystic carcinomas and mucoepidermoid carcinomas present as ulcerated painful swellings ${ }^{7}$.

Hamartomatous swellings, like osteomas, are slow growing, bony hard peripheral swellings that appear as dense radio opaque masses on radiographs ${ }^{4}$. Haemangiomas frequently appear as bluish, soft, non-fluctuant, bosselated, domed, nodular swellings on the palate and buccal mucosa ${ }^{8}$. Among cystic swellings occurring in the maxillary region, the most common are dentigerous cysts associated with the crown of unerupted or developing teeth, mostly maxillary canines, and third molars in the maxillary jaw. These are seen as slowly expanding cystic swellings with egg shell crackling of the

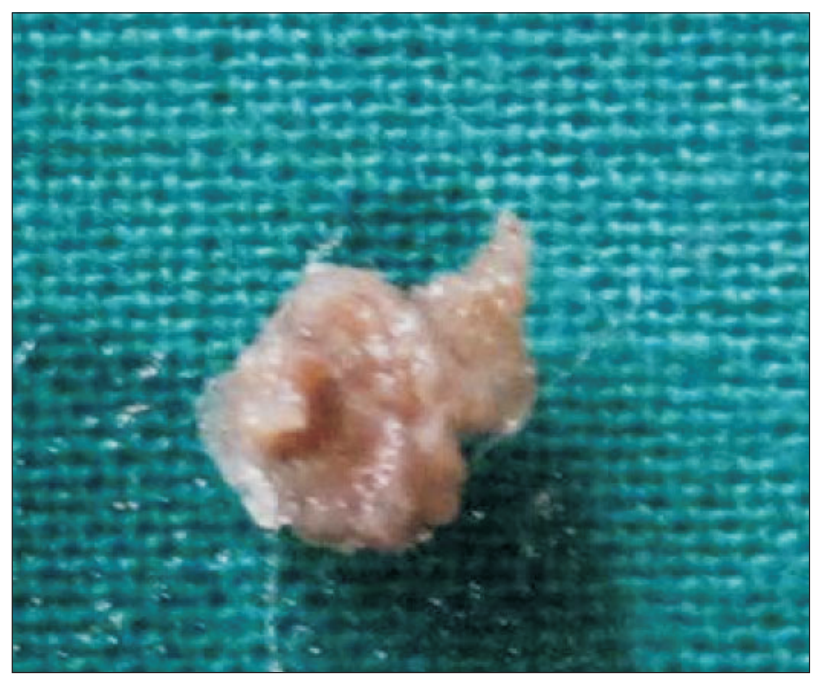

Fig. 6. Gross specimen from the lesion.

Puneet Bhargava et al: A swelling of the maxilla: a case report and differential diagnosis. J Korean Assoc Oral Maxillofac Surg 2014

cortical plates or erosion in advanced cases ${ }^{9}$. Benign lesions such as central giant cell granulomas are painless, slowly expanding swellings that have a predilection for the anterior mandibular region; they are mostly seen in younger females. Radiographically, the cyst in this study presented as a multilocular cystic swelling with fine, curved, wispy trabeculae ${ }^{10}$. Fibro osseous lesions such as fibrous dysplasia present as painless, fusiform, smoothly contoured expansion of jaw bone mostly seen in the molar and premolar regions of the maxilla rather than mandible. Radiography of fibro osseous lesions shows classical ground glass or orange peel appearance $^{11}$. OFs are indolent or aggressive well defined bony swellings mostly affecting young adult males. Mandibular lesions are seen inferior to premolars and molars and superior to the inferior alveolar canal, while maxillary lesions are seen in canine fossa and zygomatic arch areas. Radiographically, most lesions are seen as mixed radiolucent and radio dense areas with abnormal wispy and trabecular patterns of bone with a thin radiolucent line representing a fibrous capsule separating it from the surrounding bone ${ }^{12}$. Odontogenic tumors such as ameloblastoma are painless, slow growing, expansile, locally invasive swellings causing crepitus due to cortical erosion and are most common in mandibular molar ramus regions and maxillary third molar regions. Radiographs show multilocular soap bubble or honey comb patterns with coarse curved trabeculae causing extensive root resorption and regional tooth displacement ${ }^{13}$. Another common odontogenic tumor is the odontogenic myxoma, which is a painless expansion of the jaw with mobility or migration of 
regional teeth. These are situated in tooth bearing areas of the jaw, with a predilection towards older females, mostly occurring in the ramus and premolar regions of the mandible. Radiographs show a multilocular tennis racket appearance with straight trabeculae directed at right angles to one another. Osteosarcomas are one of the most common malignancy of the jaw bone, second only to multiple myeloma. Osteosarcomas present as intermittent painful swellings, with paresthesia, tooth mobility, bleeding, and asymmetry of the jaws. Chronic lesions may get ulcerated. They are mostly seen in the body of the mandible and alveolar ridge of the maxilla. Radiographs show radiolucent lesions with ragged, poorly defined borders and a band-like widening of the periodontal space ${ }^{14}$.

The term OF is used if the predominant component is bone, while cementifying fibroma is defined by the presence of curvilinear trabecular structures or spherical calcifications. In turn, lesions characterized by the presence of bone and cementum are referred to as cement OFs. When the lesion occurs in individuals between 5 and 15 years of age, it is called a juvenile $\mathrm{OF}^{1}$. Regezi et al. ${ }^{15}$ and Sciubba commented that multiple OFs are sporadic, but there is a familial inclination in some cases. Pimenta et al. ${ }^{16}$ first demonstrated sporadic and germ line mutations of the tumor suppressor gene, HRPT2, that encodes a parafibromin protein in OFs. HRPT2 generally occurs between the second and fourth decade of life, with a $1: 5$ (male : female) ratio. The most frequent location of these tumors is in the mandible involving the premolar and molar regions; the facial sinuses and nasal cavities are rarer locations ${ }^{17}$. These tumors are slow-growing, progressive, and have painless bone tumefaction. They can occasionally push the dental organs without leading to rhizolysis or altering the vitality of the adjacent teeth. The radiological images most often associated with OFs correspond to a welldefined radiolucency with or without a sclerotic margin and are often accompanied by cortical expansion. In the type that contains mainly radiopaque foci, the pattern may be similar to that seen in fibrous dysplasia, or a wispy or flocculent pattern may be seen ${ }^{18}$. The trabeculae are more radial compared to the center of the image, but, as it nears completion, a nearly total opacity of the lesion can be observed. These tumors exhibit centrifugal growth patterns rather than linear ones; therefore, the lesions grow by expansion in all directions and present as round masses. This tumor maintains a spherical shape and expands the surrounding cortical bone without cortical perforation. The lamina dura of involved teeth is usually missing, and resorption of teeth may occur. On computed radiography a well-differentiated mass with the same den- sity as bone is found, and the center of the lesion shows low density $^{17}$. OFs consist of fibrous tissues that exhibit varying degrees of cellularity and contain mineralized materials. The hard tissue portion may be in the form of trabeculae of osteoid and bone or basophilic and poorly cellular spherules that resemble cementum. Admixtures of the two types are typical. The bony trabeculae vary in size and frequently demonstrate a mixture of woven and lamellar patterns. Peripheral osteoid and osteoblastic rimming are usually present. The spherules of cementum-like material often demonstrate peripheral brush borders that blend into the adjacent connective tissue ${ }^{1}$. Enucleations or wider resection with or without reconstruction can be carried out. Resection of the peritumoral bone may have to be performed in the subperiosteal plane, which is free from lesional proliferation; preserving it facilitates later reconstruction with bone grafting. Recurrences of OFs are variable $10 \%$ to $28 \%$ after enucleation and $5 \%$ after resection $^{19}$.

The present case highlights a rare clinical presentation of OF of the maxillary tuberosity region. Swellings of the maxillary jaw may present in various ways. It is essential for physicians to assess and evaluate these lesions by differentiating between the various swellings in the maxilla. Etiology may range from traumatic to malignant.

\section{Conflict of Interest}

No potential conflict of interest relevant to this article was reported.

\section{References}

1. Gondivkar SM, Gadbail AR, Chole R, Parikh RV, Balsaraf S. Ossifying fibroma of the jaws: report of two cases and literature review. Oral Oncol 2011;47:804-9.

2. Waldron CA. Fibro-osseous lesions of the jaws. J Oral Maxillofac Surg 1993;51:828-35.

3. Wood NK, Goaz PW. Differential diagnosis of oral and maxillofacial lesions. 5th ed. St. Loius: Mosby; 1998.

4. White SC, Pharoah MJ. Diseases of bone manifested in the jaws. In: White SC, Pharoah MJ, editors. Oral radiology: principles and interpretation. 5th ed. St. Loius: Mosby; 2004:412.

5. Orpe EC, Lee L, Pharoah MJ. A radiological analysis of chronic sclerosing osteomyelitis of the mandible. Dentomaxillofac Radiol 1996;25:125-9.

6. Priddy RW. Inflammatory hyperplasias of the oral mucosa. J Can Dent Assoc 1992;58:311-5, 319-21.

7. Greenburg MS, Glick M, Ship JA. Salivary gland diseases. Burkitts oral medicine. 11th ed. Hamilton: BC Decker; 2008:217.

8. Stal S, Hamilton S, Spira M. Hemangiomas, lymphangiomas, and vascular malformations of the head and neck. Otolaryngol Clin North Am 1986;19:769-96.

9. Daley TD, Wysocki GP. The small dentigerous cyst. A diagnostic 
dilemma. Oral Surg Oral Med Oral Pathol Oral Radiol Endod 1995;79:77-81.

10. Waldron CA, Shafer WG. The central giant cell reparative granuloma of the jaws. An analysis of 38 cases. Am J Clin Pathol 1966;45:437-47.

11. Obisesan AA, Lagundoye SB, Daramola JO, Ajagbe HA, Oluwasanmi JO. The radiologic features of fibrous dysplasia of the craniofacial bones. Oral Surg Oral Med Oral Pathol 1977;44:94959.

12. Eversole LR, Merrell PW, Strub D. Radiographic characteristics of central ossifying fibroma. Oral Surg Oral Med Oral Pathol 1985;59:522-7.

13. Weissman JL, Snyderman CH, Yousem SA, Curtin HD. Ameloblastoma of the maxilla: CT and MR appearance. AJNR Am J Neuroradiol 1993; 14:223-6.

14. Clark JL, Unni KK, Dahlin DC, Devine KD. Osteosarcoma of the jaw. Cancer 1983;51:2311-6.
15. Regezi JA, Sciubba JJ, Jordan RCK. Oral pathology: clinical pathologic correlations. 5th ed. St. Louis: Saunders; 2007.

16. Pimenta FJ, Gontijo Silveira LF, Tavares GC, Silva AC, Perdigão $\mathrm{PF}$, Castro WH, et al. HRPT2 gene alterations in ossifying fibroma of the jaws. Oral Oncol 2006;42:735-9.

17. Oukabli M, Akhaddar A, Qamouss O, Chahdi H, Rimani M, Albouzidi A. Nasoethmoidal psammomatoid cemento-ossifiying fibroma with intraorbital extension. Rev Stomatol Chir Maxillofac 2010;111:43-5.

18. Su L, Weathers DR, Waldron CA. Distinguishing features of focal cemento-osseous dysplasia and cemento-ossifying fibromas. II. A clinical and radiologic spectrum of 316 cases. Oral Surg Oral Med Oral Pathol Oral Radiol Endod 1997;84:540-9.

19. Trijolet JP, Parmentier J, Sury F, Goga D, Mejean N, Laure B. Cemento-ossifying fibroma of the mandible. Eur Ann Otorhinolaryngol Head Neck Dis 2011;128:30-3. 